\title{
From Nominal To Robust Planning: The Plate-Ball Manipulation System
}

\author{
Giuseppe Oriolo* Marilena Vendittelli* Alessia Marigo ${ }^{\diamond}$ Antonio Bicchi ${ }^{\dagger}$ \\ * Dipartimento di Informatica e Sistemistica, Università di Roma "La Sapienza" \\ Via Eudossiana 18, 00184 Roma, Italy, \{oriolo, vendittelli\}@dis .uniroma1.it \\ $\diamond$ IAC-CNR, Viale del Policlinico 137, 00161 Roma, Italy, marigo@iac.rm.cnr.it \\ † Interdept. Research Center "Enrico Piaggio", Università di Pisa \\ Via Diotisalvi 2, 56100 Pisa, Italy, bicchi@ing.unipi.it
}

\begin{abstract}
Robotic manipulation by rolling contacts is an appealing method for achieving dexterity with relatively simple hardware. While there exist techniques for planning motions of rigid bodies in rolling contact under nominal conditions, an inescapable challenge is the design of robust controllers of provable performance in the presence of model perturbations. As a preliminary step in this direction, we present in this paper an iterative robust planner of arbitrary accuracy for the plateball manipulation system subject to perturbations on the sphere radius. The basic tool is an exact geometric planner for the nominal system, whose repeated application guarantees the desired robustness property on the basis of the Iterative Steering paradigm. Simulation results under perturbed conditions show the effectiveness of the method.
\end{abstract}

\section{Introduction}

Rolling manipulation has recently attracted the interest of robotic researchers as a convenient way to design dextrous hands with simplified hardware (see [1, 2, 3] and the references therein). Here, dexterity indicates the capability to relocate and reorient a manipulated object by maintaining a firm grasp on it. A first prototype of a hand purposefully implementing rolling manipulation was presented in [2]. The nonholonomic nature of rolling contacts between rigid bodies guarantees the generic controllability of rolling pairs, i.e., that any two surfaces (with the only exception of surfaces that are mirror images of each other) can be arbitrarily reoriented and relocated by rolling. While such result is limited to smooth surfaces, the case of a polyhedral object to be manipulated was considered in [4].

The archetypal example of rolling manipulation is the plate-ball system $[5,6,7,8]$ : the ball (the manipu- lated object) can be brought to any contact configuration by maneuvering the upper plate (the first finger), while the lower plate (the second finger) is fixed. Despite its mechanical simplicity, the planning and control problems for this device already raise challenging theoretical issues. In fact, in addition to the wellknown limitations due to nonholonomy (essentially, the lack of smooth stabilizability), the plate-ball system is neither flat nor nilpotentizable; therefore the classical techniques (e.g., see [9]) for planning and stabilization of nonholonomic systems cannot be applied.

To this date, only the planning problem has been attacked with some success; e.g., see the algorithms in $[1,6]$. Like for any planner based on open-loop control, however, the successful execution of maneuvers is not preserved in the presence of perturbations - some sort of feedback is necessary to induce a degree of robustness. This advancement appears to be mandatory in order to fulfill the promise of rolling manipulation of providing a reliable technological solution.

The final objective of our research is to move from planning to robust stabilization by exploiting the mechanism of iteration as proposed in [10], i.e., sampling the system state and repeatedly applying the same planner at discrete instants. In addition to the simplicity of design, this general stabilization approach (IS, or Iterative Steering) has the advantage of driving the system along the predictable trajectories typical of the planner. Such feature is particularly useful in the presence of configuration space constraints, e.g., due to workspace obstacles.

A first step in the above direction was presented in [11], where we considered the problem of rolling a ball whose radius was only known up to some measurement error, and designed a robust controller for this system by iterating an approximate planner based on a nilpotent approximation of the dynamics. While sim- 
ulations showed the effectiveness of such method in rejecting the radius perturbation, only local stability was guaranteed. Moreover, the formal proof that the controller satisfied the requirements of the IS paradigm required an additional condition on the contraction rate which eventually affected the convergence speed.

Here, we retain the general strategy (IS) but we change the basic tool, i.e., the planner. In particular, by adopting an exact (for the nominal system) planner based on geometric arguments - of interest in itself - we are able to derive a scheme that drives the perturbed plate-ball system from any configuration to the desired goal with arbitrary precision. However, as will be made clear in the paper, due to the specific nature of the nominal planning algorithm, an iterative robust planner is obtained rather than a stabilization method.

The paper is organized as follows. In Sect. 2, the model of the plate-ball system is briefly described. In Sect. 3, a spinning maneuver which achieves rotation of the ball around its vertical axis is proposed in two versions: a open-loop and a closed-loop version. The exact planner for the nominal system using the latter is devised in Sect. 4, while the iterative planner generated by the IS strategy is described in Sect. 5, where we also report simulation results confirming the achieved robustness. A short discussion on the perspectives of this work concludes the paper.

\section{The model}

The plate-ball system of Fig. 1 is a special case of rolling contact between regular surfaces (see[3] for a complete treatment). Its kinematic equations describe the evolution of the (local) coordinates of the contact points on the plate, $\alpha_{p}=(x, y) \in \mathbb{R}^{2}$, and on the ball, $\alpha_{b}=(u, v) \in \mathbb{R}^{2}$, as well as of the sphere orientation $\psi$ with respect to the plane, given by the holonomy angle between the two Gauss frames associated to $\alpha_{p}$ and $\alpha_{b}$.

Denoting by $\rho$ its radius, the ball can be parameterized as

$$
f(u, v)=\left(\begin{array}{c}
\rho \cos v \cos u \\
\rho \cos v \sin u \\
\rho \sin v
\end{array}\right)
$$

with $\{(u, v) \mid-\pi<u<\pi,-\pi / 2<v<\pi / 2\}$. Following the derivation of Montana [5], one obtains the following kinematic equations

$$
\left(\begin{array}{c}
\dot{\alpha}_{p} \\
\dot{\alpha}_{b} \\
\dot{\psi}
\end{array}\right)=\left(\begin{array}{cc}
0 & 1 \\
1 & 0 \\
\frac{\cos \psi}{\rho \cos v} & -\frac{\sin \psi}{\rho \cos v} \\
-\frac{\sin \psi}{\rho} & -\frac{\cos \psi}{\rho} \\
\frac{\tan v \cos \psi}{\rho} & -\frac{\tan v \sin \psi}{\rho}
\end{array}\right) w
$$

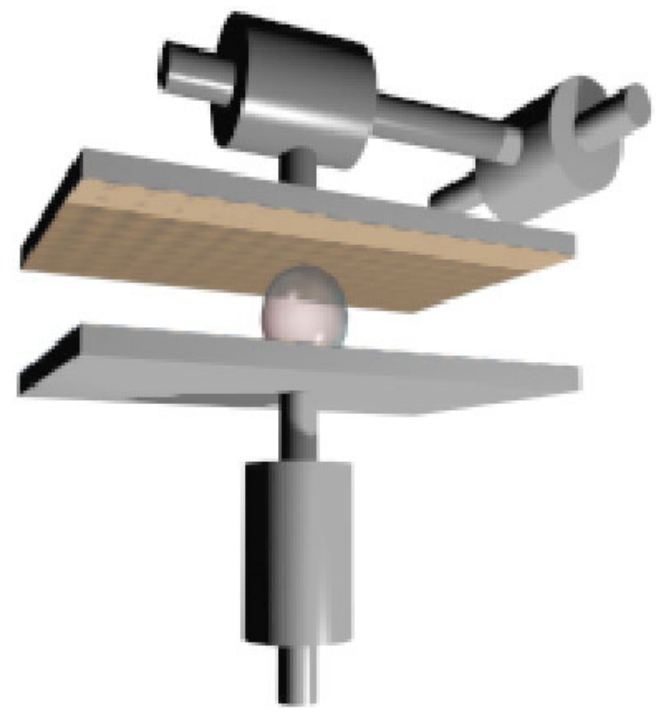

Figure 1: The plate-ball manipulation system

where $w$ is the cartesian velocity $(\dot{y}, \dot{x})$ of the contact point on the plane, which we assume to be the control input.

\section{The spinning maneuver}

The planner to be presented in Sect. 4 requires the capability of 'spinning' the ball, i.e., changing $\psi$ without altering the values of the other system coordinates. The perfect rolling assumption prevents a pure rotation of the ball around an axis which is perpendicular to the finger surface at the contact point, for this would violate the underlying nonholonomic constraint. However, due to the controllability of the system, for any angle $\Delta \psi$ there exists a control function $w(t)$ steering the system from the configuration $\left(\alpha_{p}, \alpha_{b}, \psi\right)$ to the configuration $\left(\alpha_{p}, \alpha_{b}, \psi+\Delta \psi\right)$ in finite time. In this section, we present two ways to compute such a control function: the first in open-loop, and the second - a slight modification of the first - in closed-loop. The utility of the closed-loop version in setting up our planner will be clarified in Sect. 4 .

\subsection{The open-loop maneuver}

The spinning maneuver is obtained by a sequence of three control functions. Up to a change of coordinates, we can assume that at the beginning of the maneuver the contact between the ball and the lower finger occurs at the south pole (i.e., $v=-\frac{\pi}{2}$ ).

The first control function forces the the ball to roll along a geodesic $\left(u=\right.$ constant $\left.=u_{0}\right)$ so that the con- 
tact point is steered from the south pole to the particular parallel corresponding to $v=\bar{v}$, whose determination is discussed later. This is simply obtained by the following steering control:

$$
w(t)=\left(\begin{array}{c}
\sin \psi_{0} \\
\cos \psi_{0}
\end{array}\right)
$$

where $\psi_{0}$ is the initial orientation of the ball. In order to reach $\bar{v}$, this steering control must be applied over a time interval $[0, \rho(\bar{v}+\pi / 2)]$.

The second control drives the contact point along the same parallel until the contact point on the plane completes a circle. When this happens, $u$ has reached a value $u_{0}+\Delta u$, with $\Delta u$ determined as follows.

Assuming without loss of generality $\bar{v} \in(-\pi / 2,0)$, the radius of the circle on the plane is $\rho \tan \left(\bar{v}+\frac{\pi}{2}\right)$, so that its length is $2 \pi \rho \tan \left(\bar{v}+\frac{\pi}{2}\right)$. On the other hand, the parallel traced by the contact point on the sphere is a circle of radius $\rho \sin \left(\bar{v}+\frac{\pi}{2}\right)=\rho \cos \bar{v}$. Being the length of the path traced by the contact point on the sphere equal to the length of the path traced by the contact point on the plane, we have

$$
2 \pi \rho \tan \left(\bar{v}+\frac{\pi}{2}\right)=\Delta u \rho \cos \bar{v}
$$

from which

$$
\Delta u=-\frac{2 \pi}{\sin \bar{v}}
$$

We can determine the net change $\Delta \psi$ that the sphere orientation undergoes at the end of the spinning maneuver as the integral of the gaussian curvature over the region bounded by the closed path traced by the contact point on the sphere (total curvature):

$$
\begin{aligned}
\Delta \psi & =\int_{u_{0}}^{u_{0}+\Delta u} \int_{-\frac{\pi}{2}}^{\bar{v}} K\left\|f_{u} \times f_{v}\right\| d v d u \\
& =\int_{u_{0}}^{u_{0}+\Delta u} \int_{-\frac{\pi}{2}}^{\bar{v}} \cos v d v d u=(1+\sin \bar{v}) \Delta u
\end{aligned}
$$

where $K$ is the gaussian curvature of the sphere.

Plugging the expression of $\Delta u$ in the latter equation we get

$$
\Delta \psi=-2 \pi \frac{1+\sin \bar{v}}{\sin \bar{v}}
$$

so that the parallel to be traveled in order to spin the ball by $\Delta \psi$ is identified by

$$
\sin \bar{v}=-\frac{2 \pi}{2 \pi+\Delta \psi} .
$$

It is easy to verify that a steering control realizing the rotation on the parallel is

$$
w(t)=\left(\begin{array}{c}
-\cos \left(\psi_{0}+\frac{\tan \bar{v}}{\rho} t\right) \\
\sin \left(\psi_{0}+\frac{\tan \bar{v}}{\rho} t\right)
\end{array}\right),
$$

to be applied over a time interval $[0,2 \pi \rho / \tan \bar{v}]$

The spinning maneuver is completed by a third control action (the opposite of function (2)) that simply brings the sphere back to the south pole along the geodesic $u=$ constant $=u_{0}+\Delta u$. At this point, while the contact point on the plane and on the sphere are back to the starting configuration, the orientation has been changed as desired.

\subsection{The closed-loop maneuver}

When the radius $\rho$ of the sphere is not exactly known, the spinning maneuver so far described cannot be executed. In fact, the steering controls (2) and (3) require the value of $\rho$ in their expression and/or duration. If the nominal value of $\rho$ used to compute the controls is different from the actual value, there will be three consequences: (i) the value of $v$ reached with control (2) is different from the desired $\bar{v}$, (ii) the sphere under control (3) does not roll along a parallel, and (iii) the path traced by the contact point on the plane is not a closed circle. In terms of the final configuration of the ball, this means not only that the desired orientation is not reached, but also that $u, v$, $x$ and $y$ do not go back to their initial values due in particular to (ii) and (iii).

While the error in $\psi$ is acceptable and will be recovered by the iterative version of the planner to be presented in Sect. 4, the non-cyclicity in the other variables would destroy the convergence of the planner. We therefore devise a modified version of the spinning maneuver that uses a closed-loop control to roll the ball along a parallel without knowing its radius.

Given the rolling equations (1), it is straightforward to verify that the closed-loop steering control

$$
w(t)=\left(\begin{array}{c}
-\cos \psi(t) \\
\sin \psi(t)
\end{array}\right)
$$

yields $\dot{v} \equiv 0$ (i.e., $v(t) \equiv \bar{v}$ ), driving the contact point on the sphere along the parallel reached after the application of the first control (2). The other system equations can be integrated in closed form; in particular, the other coordinates of the ball are obtained as

$$
\begin{aligned}
& u(t)=u_{0}-\frac{1}{\rho \cos \bar{v}} t \\
& \psi(t)=\psi_{0}-\frac{\tan \bar{v}}{\rho} t,
\end{aligned}
$$

while the contact point on the plane will describe the circle

$$
\begin{aligned}
& x(t)=x_{c}+\frac{\rho}{\tan \bar{v}} \sin \left(\psi_{0}+\frac{\tan \bar{v}}{\rho} t\right) \\
& y(t)=y_{c}+\frac{\rho}{\tan \bar{v}} \cos \left(\psi_{0}+\frac{\tan \bar{v}}{\rho} t\right)
\end{aligned}
$$


with radius $\rho / \tan \bar{v}$ and center in

$$
\begin{aligned}
& x_{c}=x_{0}-\frac{\rho}{\tan \bar{v}} \sin \psi_{0} \\
& y_{c}=y_{0}-\frac{\rho}{\tan \bar{v}} \cos \psi_{0} .
\end{aligned}
$$

Equation (4) indicates that the circle is completed at time $\bar{t}=\left|\frac{2 \pi \rho}{\tan \bar{v}}\right|$. Once again, the control duration time would depend on the ball radius and, hence, the 'parallel roll' is not robust yet w.r.t. perturbation on $\rho$. The desired robustness can be achieved by modifying the open-loop control (3) as follows

$$
w(t)=\left(\begin{array}{c}
-\cos \psi(t) s(\psi) \\
\sin \psi(t) s(\psi)
\end{array}\right)
$$

with

$$
s(\psi)=1-\delta_{-1}\left(\psi-\psi_{0}-2 \pi\right)
$$

where $\delta_{-1}$ is the Heaviside step function.

\section{The exact planner}

Denote by $\mathcal{M}$ the plate-ball configuration space, which is locally diffeomorphic to $\mathbb{R}^{2} \times \mathbb{R}^{2} \times S^{1}$. Let $p_{0}=$ $\left(\alpha_{p}^{0}, \alpha_{b}^{0}, \psi^{0}\right)$ and $p_{g}=\left(\alpha_{p}^{g}, \alpha_{b}^{g}, \psi^{g}\right)$ be two points in $\mathcal{M}$. The algorithm steers the system from $p_{0}$ to $p_{g}$ through the following intermediate configurations:

$$
\begin{aligned}
& p_{0} \stackrel{\text { Step } 1}{\mapsto} p_{1}=\left(\alpha_{p}^{g}, \alpha_{b}^{1}, \psi^{1}\right) \\
& p_{1} \stackrel{\text { Step } 2}{\mapsto} p_{2}=\left(\alpha_{p}^{g}, \alpha_{b}^{g}, \psi^{2}\right) \\
& p_{2} \stackrel{\text { Step } 3}{\mapsto} p_{g}=\left(\alpha_{p}^{g}, \alpha_{b}^{g}, \psi^{g}\right)
\end{aligned}
$$

Step 1 is simply executed by applying a constant control such that the velocity of $\alpha_{p}$ (the contact point on the plane) is a vector with the same direction of $\left(\alpha_{p}^{g}-\alpha_{p}^{0}\right)$ and unit norm:

$$
w(t)=\frac{\left(\alpha_{p}^{g}-\alpha_{p}^{0}\right)}{\left\|\alpha_{p}^{g}-\alpha_{p}^{0}\right\|}, \quad t \in\left[0, T_{1}\right], \quad T_{1}=\left\|\alpha_{p}^{g}-\alpha_{p}^{0}\right\| .
$$

Step 2 is performed by alternating two maneuvers. Up to a change of coordinates, assume again that the contact point on the sphere reached at the end of Step 1 is the south pole. The first maneuver, realized by a constant control of the form

$$
w(t)=w_{u}, \quad t \in\left[0, T_{2}\right], T_{2}=\frac{1}{4}\left|v^{g}+\frac{\pi}{2}\right| \rho,\left\|w_{u}\right\|=1,
$$

rolls the ball along an arc of the geodesic corresponding to $u_{g}$, which joins the initial and the desired contact point on the sphere. The second is a closed-loop spinning maneuver that rotates the ball of $\frac{\pi}{2}$ around the axis perpendicular to the finger through the point of contact as explained in Sect. 3.2. By repeating four times the two maneuvers in sequence, the contact point on the ball is steered to $\alpha_{b}^{g}$ while the contact point on the plane has come back to the initial point $\alpha_{p}^{g}$, having traced a square of edge $T_{2}$.

Step 3 brings the last variable $\psi$ to its desired value by using the closed-loop spinning maneuver to achieve a rotation $\Delta \psi=\psi^{g}-\psi^{2}$.

\section{$5 \quad$ Robust planning by IS}

As mentioned in the introduction, our idea is to robustify the planner by using the iteration mechanism. The theoretical framework of IS [10] indicates that a robust stabilizer can be obtained by iterating a planner with suitable properties, the most relevant of which is (Hölder-)continuity at the origin with respect to the desired reconfiguration. In practice, this property means that the configuration space path generated by the planner 'shrinks' and eventually vanishes when the desired reconfiguration goes to zero.

Without going into technical details, it is clear that the planner of the previous section does not possess this property ${ }^{1}$, due to the repeated spinning maneuvers in Step 2, each of which adds $\pi / 2$ to the current value of $\psi$ by driving $x, y$ along the same path on the plane (see Fig. 4). Therefore, the simple iteration of the whole planner does not yield a robust stabilizer.

However, an arbitrarily accurate robust planner can be obtained by iterating separately Steps 2 and 3 of the planner (note that Step 1 is insensitive to perturbations on the sphere radius) until the state error is below a given tolerance. The proof of convergence of the error with the iterations is lengthy and therefore omitted, but basically relies on a simple property of perturbed discrete-time systems [10, Lemma 1]. The same proof guarantees that the steering error converges exponentially to zero starting from any configuration.

We note that the adoption of the closed-loop version of the spinning maneuver in the planner is essential for guaranteeing robustness. In fact, if the sphere radius is not exactly known, the non-cyclicity in $u, v, x$ and $y$ of the open-loop spinning maneuver pointed out at the beginning of Sect. 3.2 would result in a persistent perturbation on the sampled error dynamics, which would destroy the convergence. All the other induced perturbations are instead non-persistent, and therefore rejected by the iteration mechanism itself.

To show the effectiveness of the iterative planner, we report the results of the simulated execution of a

\footnotetext{
${ }^{1}$ Actually, the same is true for any other exact planner in the literature; instead, the approximate planner used in [11] satisfies the continuity property.
} 


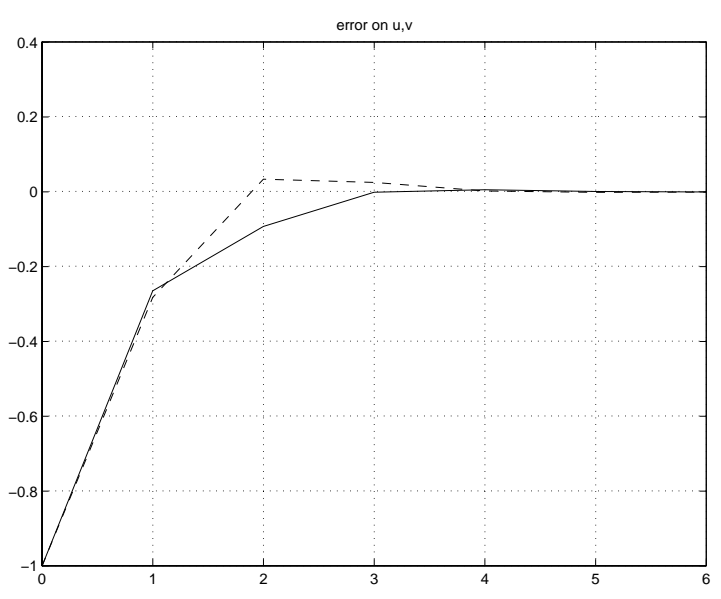

Figure 2: Error on $u$ (solid) and $v$ (dashed) at the end of each iteration of Step 2

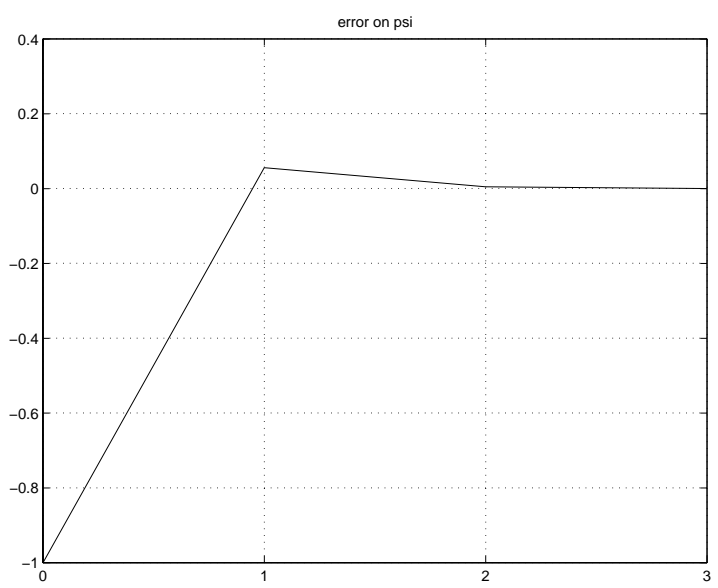

Figure 3: Error on $\psi$ at the end of each iteration of Step 3

steering task. The system has to reach the origin of the configuration space starting from the configuration $(1,1,1,1,1)(\mathrm{m}, \mathrm{m}, \mathrm{rad}, \mathrm{rad}, \mathrm{rad})$, despite a $5 \%$ perturbation on the nominal unit radius. The iteration of Steps 2 and 3 is interrupted as soon as the norm of the corresponding error (on $u, v$ and $\psi$, respectively) is below $10^{-3}$. Figure 2 reports the values of the error on $u, v$, during the iterations of Step 2, while Fig. 3 refers to error on $\psi$ during the iterations of Step 3. Figures 4 7 show four paths of the contact point on the plane, corresponding respectively to Step $1+$ the first iteration of Step 2, the third iteration of Step 2, the first iteration of Step 3, and the third iteration of Step 3. Note that the path on the plane contracts during the repeated execution of Step 3.

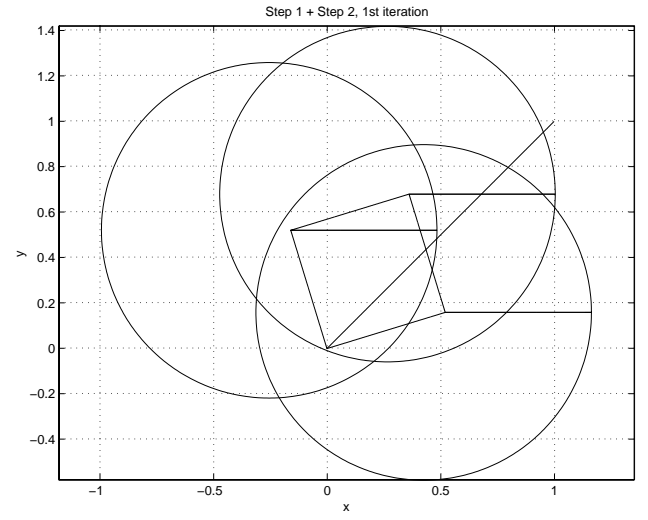

Figure 4: Path of the contact point on the plane during Step 1 and the first iteration of Step 2

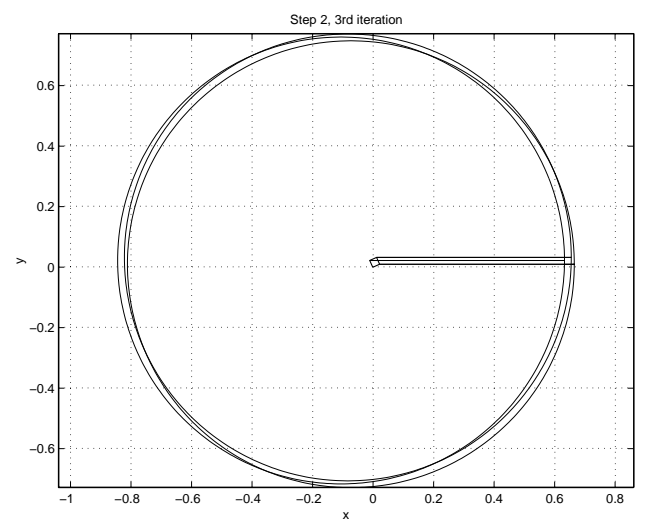

Figure 5: Path of the contact point on the plane during the third iteration of Step 2

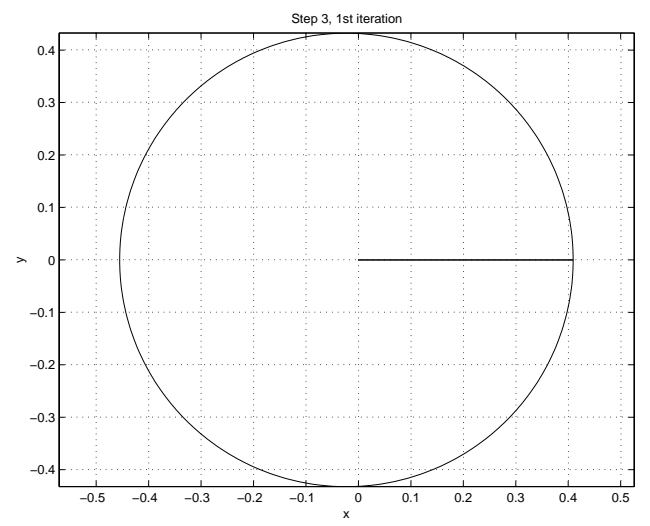

Figure 6: Path of the contact point on the plane during the first iteration of Step 3 


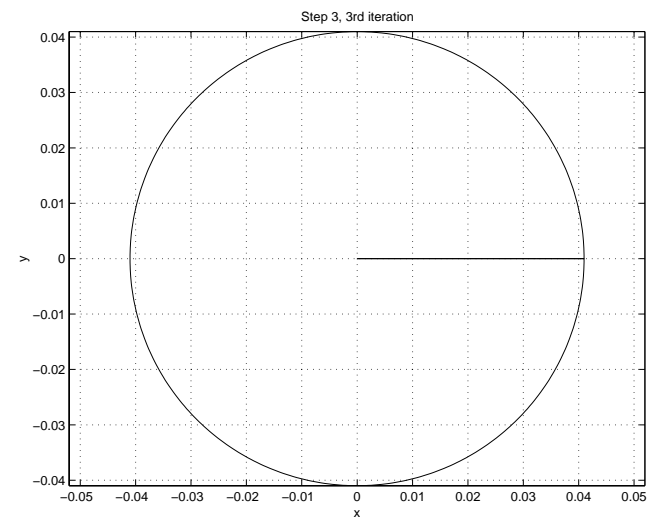

Figure 7: Path of the contact point on the plane during the third iteration of Step 3

\section{Conclusions}

As an intermediate result of our investigation aimed at deriving robust controllers for rolling manipulation mechanisms, we have presented a planner that can drive the plate-ball system to a desired configuration with arbitrary precision in spite of perturbations on the sphere radius. The planner relies on the repeated application of a steering algorithm that is exact for the nominal system and has been designed to guarantee its effectiveness within the iterative framework.

Future work on this planner includes the computation of explicit bounds on the admissible perturbation as well as its combination with a motion planning scheme to ensure the successful execution of maneuvers in the presence of obstacle. At a more general level, however, the achievement of our long-term objective (robust stabilization) will pass through the synthesis of nominal planners with the analytic properties required by the IS paradigm (Hölder-continuity of the steering control law with respect to the desired reconfiguration). In this respect, the so-called locallocal property of [12] and topological property of [13] (of which the former is a relaxation) are of interest because they represent the topological counterpart of the Hölder-continuity condition. Therefore, it will be necessary to bridge steering controllers and geometrical planners by transferring algorithms and properties between them.

\section{Acknowledgments}

Work supported by the following contracts: MURST MISTRAL, IST-2001-37170 (RECSYS), IST-200138040 (TOUCH-HAPSYS), ASI I/R/124/02 (TEMA).

\section{References}

[1] A. Marigo and A. Bicchi, "Rolling bodies with regular surface: controllability theory and applications," IEEE Trans. on Automatic Control, vol. 45, pp. 1586$1599,2000$.

[2] A. Bicchi and R. Sorrentino, "Dexterous manipulation through rolling," 1995 IEEE Int. Conf. on Robotics and Automation, pp. 452-457, 1995.

[3] R. M. Murray, Z. Li, and S. S. Sastry, A Mathematical Introduction to Robotic Manipulation, CRC Press, 1994.

[4] A. Marigo, Y. Chitour, and A. Bicchi, "Manipulation of polyhedral parts by rolling," IEEE Int. Conf. on Robotics and Automation, pp. 2992-2997, 1997.

[5] D. J. Montana, "The kinematics of contact and grasp," Int. J. of Robotics Research, vol. 7, no. 3, pp. 17-32, 1988.

[6] Z. Li and J. Canny, "Motion of two rigid bodies with rolling constraint," IEEE Trans. on Robotics and Automation, vol. 6, pp. 62-72, 1990.

[7] V. Jurdjevic, "The geometry of the plate-ball problem," Arch. for Rational Mechanics and Analysis, vol. 124, pp. 305-328, 1993.

[8] R. W. Brockett and L. Dai, "Non-holonomic kinematics and the role of elliptic functions in constructive controllability," in Nonholonomic motion planning, Z. Li and J. F. Canny, Eds., pp. 1-21. Kluwer Academic Publishers, 1993.

[9] J.-P. Laumond (Ed.), Robot Motion Planning and Control, Springer-Verlag, 1998.

[10] P. Lucibello and G. Oriolo, "Robust stabilization via iterative state steering with an application to chainedform systems," Automatica, vol. 37, pp. 71-79, 2001.

[11] G. Oriolo and M. Vendittelli, "Robust stabilization of the plate-ball manipulation system," 2001 IEEE Int. Conf. on Robotics and Automation, pp. 91-96, 2001.

[12] A. Marigo and A. Bicchi, "A local-local planning algorithm for rolling objects," 2002 IEEE Int. Conf. on Robotics and Automation, pp. 1759-1764, 2002.

[13] S. Sekhavat and J. P. Laumond, "Topological properties for collision free nonholonomic motion planning: the case of sinusoidal inputs for chained form systems," IEEE Trans. on Robotics and Automation, vol. 14, no. 5, pp. 671-680, 1998. 